\title{
Pollen Pistil Interaction in the Ovary in Fruit Trees
}

\author{
Herrero, M., Arbeloa, A., Gascon, M. \\ Unidad de Fruticultura \\ CENIA DE AUIa Dei \\ Apartado 727 \\ Zaragoza (Spain)
}

\section{Introduction}

Siphonogamy, or the fact that fertilization is mediated through a pollen tube, is emerging as a uniquely adapted system for male-female interaction (Heslop-Harrison, 1983) and for male gametophyte selection (Mulcahy, 1979). However, we still know very little on the physiological mechanisms that control such selection. A better understanding of male-female interaction from pollination to fertilization may enlighten this process. In this respect most of the work has been done regarding pollen germination at the stigma (Heslop-Harrison and Heslop-Harrison, 1985 ) or concerning pollen tube growth in the style (van Went and willemsen, 1984). However, there is a paucity of data regarding pollen tube growth in the ovary region (Knox, 1984).

It has been tacitly assumed that, once the pollen tubes enter the ovary, fertilization was an straightforward process. However, working with fruit tree species, we have observed that in the ovary a number of interactive processes are set up from the arrival of the pollen tubes until fertilization occurs. In this paper we discuss these events taking place in the ovary and their possible implications in male-female interaction and in gametophyte selection.

\section{The effect of the ovary on pollen tube growth}

In a recent study carried out in peach (Herrero and Arbeloa, 1988), we have observed that a variable lapse of time occurs from the arrival of the pollen tubes at the base of the style until fertilization takes place. This time may be longer than the time required by the pollen tubes to travel down the style.

When the pollen tubes reach the base of the style and enter 
the ovary they meet the obturator that is a placental protuberance connecting the style with the ovule micropyle. An histochemical study of this structure (Arbeloa and Herrero, 1987) reveals that when the pollen tubes arrive at the obturator they stop and growth is not resumed until five days later. On the arrival of the pollen tubes the obturator cells are full of starch reserves. Five days later starch fades from these cells and a secretion that stains for carbohydrates and for proteins is produced. Concomitantly with the production of this secretion growth of the pollen tubes is resumed on it. The fact that pollen tubes require this secretion to grow reinforces the idea that pollen tube growth along the pistil is heterotrophic (Herrero and Dickinson, 1979, Herrero and Arbeloa, 1938). However a major difference exists between growth in the style and on the obturator. While in the transmitting tissue starch digestion is triggered by pollination and only occurs in compatible matings (Herrero and Dickinson 1979), in the obturator this process is independent of pollination and appears to be a maturative stage of the pistil for it takes place in a similar way in pollinated than in unpollinated flowers (Arbeloa and Herrero, 1987).

Once the pollen tubes have passed along the obturator, callose starts to accumulate on this structure (Arbeloa and Herrero, 1987). This mechanism confers the obturator a critical role in controlling pollen tube penetration into the ovary since it acts as a leaving bridge either connecting or isolating the ovary to the style. Thus, pollen tube growth is not possible before the secretion phase, neither is it possible later once the obturator degenerates.

It has been observed that pollen tubes grow on the obturator in a number of unrelated species (Tilton and Horner, 1980; Tilton et a 1., 1984; Hill and Lord, 1987). However, the fact that growth of the pollen tubes on the obturator is controlled by means of a discontinuous secretion has been, so far, only observed in peaches (Arbeloa and Herrero, 1987). It may be worth to evaluate if this process is extensible to other species and if in these species the obturator may also be a physical structure that supports male female interaction and that regulates pollen tube passage to the ovule. 